C. W. COLEY, D. A. THOMAS, III, J. A. M. LUMMISS, J. N. JAWORSKI, C. P. BREEN,

V. SCHULTZ, T. HART, J. S. FISHMAN, L. ROGERS, H. GAO, R. W. HICKLIN, P. P. PLEHIERS, J. BYINGTON, J. S. PIOTTI, W. H. GREEN, A. J. HART, T. F. JAMISON*, K. F. JENSEN*

(MASSACHUSETTS INSTITUTE OF TECHNOLOGY, CAMBRIDGE, USA)

A Robotic Platform for Flow Synthesis of Organic Compounds Informed by AI Planning

Science 2019, 365, DOI: 10.1126/science.aax1566.

\title{
Automation of Flow Syntheses by Al Synthetic Route Design and Implementation
}

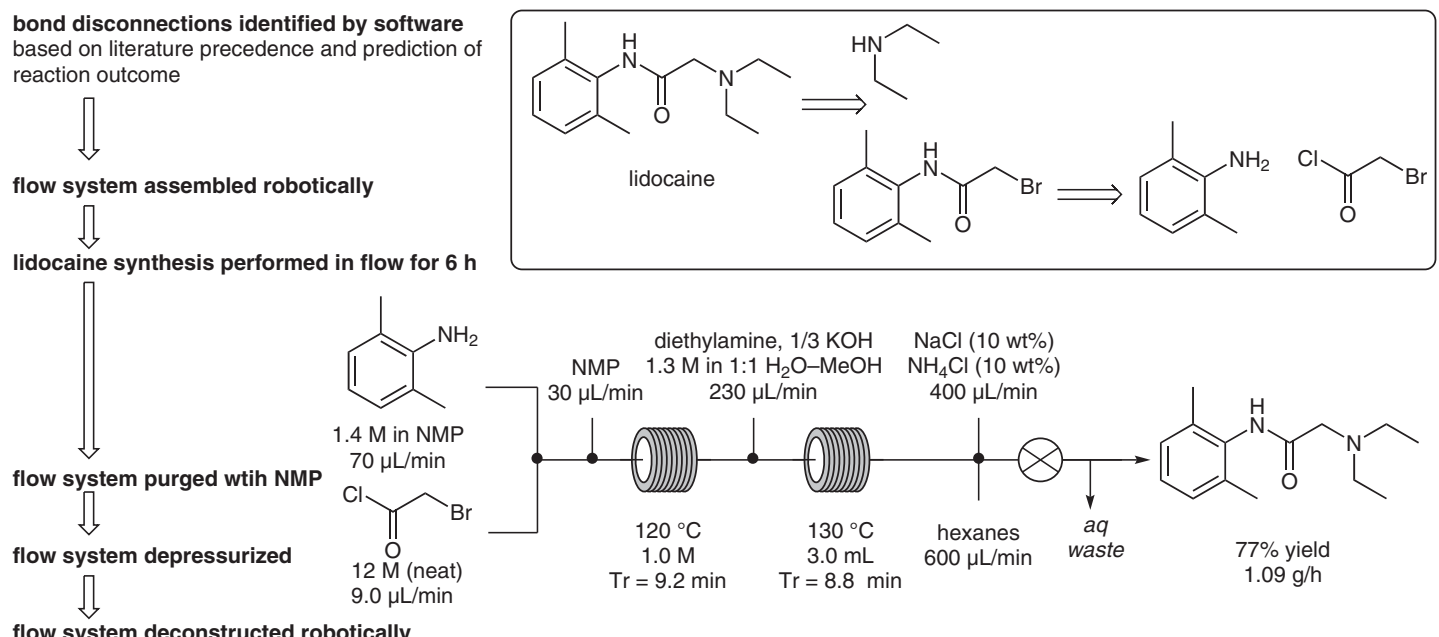

Significance: This paper details a remarkable achievement in the synthesis of complex drug molecules by using artificial intelligence (AI) and robotics. In this process, synthetic route selection is performed by using Al-aided synthesis planning and, after revision by experienced organic chemists, is carried out on a robotic platform in which flow systems are assembled, processes are run, and the reactors are disassembled and stored. This paper demonstrates this process for 15 small drug molecules (e.g., lidocaine, as shown above). Some interesting components of the planning process include the ability of the software to predict side reactions and to flag potentially complicated reaction mixtures due to incompatibility or chemoselectivity issues, as well as the ability to choose a reagent from various options on the basis of the predicted confidence that the reagent will provide the best outcome.
Comment: One challenge acknowledged by the authors is the direct translation of batch processes from patent and literature reports into continuous processing, which requires the external influence of a chemist to evaluate the process for performance in flow (stoichiometry, solubility, impurities). As new processes are enabled by flow technologies, a richer database for reactions that are compatible for this technology will become available, and this might remove the need for human intervention during route optimization and definition of the chemical recipe files, permitting rapid transfer of synthetic routes to the automated robotic platform.

\section{Key words}

artificial intelligence

reaction design

flow synthesis

automated synthesis

robot synthesizer 